\title{
A selective vacuum assisted mesh mediated fascial traction approach following temporary abdominal containment for trauma laparotomy is effective in achieving closure
}

\author{
CJ Steenkamp ${ }^{1}$, VY Kong ${ }^{1}$, JL Bruce ${ }^{1}$, GL Laing ${ }^{1}$, DL Clarke ${ }^{2}$ \\ ${ }^{1}$ Pietermaritzburg Metropolitan Trauma Service, Department of Surgery, University of KwaZulu Natal, Durban, South Africa \\ ${ }^{2}$ Department of Surgery, University of the Witwatersrand, Johannesburg, South Africa
}

Corresponding author: Christien Steenkamp (christiensteenkamp@yahoo.co.uk)

\begin{abstract}
Background: Definitive primary abdominal closure is often not possible nor desirable following trauma laparotomy. In such situations, temporary abdominal containment (TAC) is necessary. This audit reviews our experience with TAC and interrogates our use of the Vacuum Assisted Mesh Mediated Fascial Traction approach (VAMMFT) to achieve delayed closure of the Open Abdomen (OA).

Methods: We conducted a retrospective study over a 4-year period of trauma patients who underwent a trauma laparotomy and who required a TAC.

Results: Over the four-year period, 596 patients underwent a laparotomy for trauma. Of these trauma laparotomies, $463(78 \%)$ underwent primary closure and $133(22 \%)$ required a TAC. Of these 133 patients who required a TAC, 37 died, 41 underwent delayed primary fascial closure at repeat laparotomy and 55 were left with an OA. Of this cohort of 55 patients, 15 underwent a VAMMFT procedure. The VAMMFT procedure yielded a $60 \%$ closure rate, with failure to close being due to late mesh insertion and sepsis.

Conclusion: Our initial results with VAMMFT are encouraging. The technique appears to be effective and safe. Ongoing audit will allow us to accrue more patients and to better refine our algorithms and strategies.

Key words: Vacuum assisted closure, Surgical Meshes, Occlusive dressings, Surgical Closure Techniques, Ventral hernia.
\end{abstract}

\section{Introduction:}

Definitive primary abdominal closure is preferred for all abdominal surgery; however, it has been increasingly recognised that primary closure is often not possible nor desirable following trauma laparotomy. ${ }^{1-3}$ In such situations temporary abdominal containment (TAC) is necessary., ${ }^{4,5}$ If delayed primary closure cannot be obtained quickly and efficaciously, the patient is left with an open abdomen (OA), which is a morbid condition. With the increased recognition of the need for TAC there has been increased interest in the management of the OA. ${ }^{5,6}$ Initially TAC was achieved by the use of low cost, sterile plastic being sutured to either the sheath or to the skin. This became known colloquially as the Bogota bag. ${ }^{4,5,6}$ The Bogota bag, however, had a number of limitations. It allowed the sheath to retract, making it difficult to close the abdomen at a later stage, and allowed intraabdominal fluid to egress freely so necessitating frequent dressing changes. In addition, patients in whom a Bogota bag was applied were frequently left with a large ventral hernia.
In response to these problems, two broad strategies have been employed to manage the OA and to try and achieve delayed closure. These are traction type devices designed to pull the edges of the fascial sheath together and vacuum type devices designed to facilitate fascial closure and remove egressing fluids from the wound. ${ }^{7,8}$ The development of sophisticated vacuum assist closure devices has allowed for better wound management and for better closure rates according to a number of authors. ${ }^{9,10,11,12,13}$ A further technique has been described which combines both negative pressure and traction and this is known as the Vacuum Assisted Mesh Mediated Fascial Traction (VAMMFT) approach..$^{14,15}$ Our institution has used a modified VAMMFT approach for the last two years. In light of this, we set out to audit our experience with the OA and with our modified VAMMFT approach. We hoped to benchmark our outcomes and to use this data to refine our current management algorithms and strategies. In Table 1, we compare different closure techniques and closure rates, with VAMMFT. As our protocol is refined, improved closure rates should be achieved. 


\section{Clinical Setting}

The study was based at the Pietermaritzburg Metropolitan Trauma Service (PMTS), Pietermaritzburg, South Africa, and focused on trauma patients who had an OA, as part of their care at our institution. The PMTS provides definitive trauma service to the city of Pietermaritzburg, and parts of KwaZulu Natal (KZN) province. The PMTS maintains a formal regional trauma registry (HMER). All patients who present to our trauma center are prospectively entered into the database, and the information entered includes details regarding injury mechanism, operative intervention, patient progress and clinical outcomes. Ethics approval for the maintenance of this registry for both clinical care and research has been formally endorsed by the Biomedical Research Ethics Committee (BREC) of the University of Kwa-Zulu Natal (UKZN). The ethics number is BCA 221/13.

\section{Methods}

The HEMR was retrospectively reviewed for the four-year period December 2012 to December 2016 and all patients who required an $\mathrm{OA}$ following a trauma laparotomy were identified and reviewed.

\section{Terms and Descriptions}

We use the following definitions when discussing the $\mathrm{OA}$ in our institution.

- Primary Closure: refers to definitive closure of the sheath and fascia following the index laparotomy.

- Primary Temporary Abdominal Containment (TAC): refers to the use of a TAC device following the index laparotomy.

- Delayed primary fascial closure: refers to fascial closure achieved in hospital at repeat laparotomy without recourse to a device.

- Secondary Closure: refers to all patients who had had an open abdomen closed prior to discharge from hospital with the assistance of a device.

- Open Abdomen: This describes the patient who has required TAC and whose fascial edges are not yet approximated.
- Ventral hernia: refers to all patients who leave hospital with a primary fascial defect covered by a skin graft or granulation tissue which requires delayed closure at a future date.

\section{Management}

All patients who require emergency laparotomy for trauma are resuscitated and expedited to the operating room. TAC is indicated for all damage control operations and all patients where primary closure is not technically possible due to loss of abdominal wall substance or due to gross abdominal swelling and physiological compromise. If TAC is required, the socalled "Bogota bag" approach is used. A plastic vacolitre bag is opened and sutured with a continuous large nylon suture to the skin. A vacuum dressing is applied using suction catheters which have side-holes cut in them. The catheters are applied to the surface of the bag and secured with an occlusive transparent dressing. Wall suction is applied at $50 \mathrm{~mm} \mathrm{Hg}$. The suction functions by removing effluent that egresses from the wound and abdominal cavity and is placed on top of the Bogota bag to prevent direct suction on bowel and increasing the risk of fistula formation. The Bogota bag approach is used in our institution as it appears to be more robust and durable in our environment than the Opsite sandwich, which is associated with a risk of evisceration during patient movement.

Once a patient has undergone a TAC, we return to the operating room within 48 hours with the intention of achieving delayed primary closure. If delayed primary closure is not possible then the patient per definition has an OA. Prior to the introduction of VAMMFT management was individualised. If delayed primary closure could not be achieved within a week then the wound was allowed to granulate. This resulted in a large ventral hernia. Since January 2016 we have adopted the VAMMFT approach as described by Rasilainen and colleagues, ${ }^{14}$ to patients with an OA.

\section{VAMMFT}

This uses, a commercial vacuum-assisted wound closure system (V.A.C. Abdominal Dressing System; KCI, San Antonio, Texas, USA) in conjunction with a non-absorbable

\begin{tabular}{|c|c|c|c|c|c|c|c|c|}
\hline Author & Year & Technique & $\begin{array}{c}\text { Traction } \\
\text { sutures }(\mathbf{y} / \mathbf{n})\end{array}$ & $\begin{array}{c}\text { Vacuum- } \\
\text { assisted } \\
(\mathbf{y} / \mathbf{n}) \\
\end{array}$ & $\operatorname{Mesh}(\mathbf{y} / \mathbf{n})$ & $\begin{array}{l}\text { Fistula } \\
\text { rate }\end{array}$ & $\begin{array}{c}\text { Fascial } \\
\text { closure rate }\end{array}$ & Complications (other) \\
\hline Rasilainen $^{14}$ & 2012 & $\begin{array}{l}\text { Vacuum and } \\
\text { mesh mediated } \\
\text { fascial traction }\end{array}$ & Y - sheath & Y & $\begin{array}{l}\text { Polypropolene } \\
\text { mesh }\end{array}$ & $12 \%$ & $78 \%$ & 35.6\% Mortality \\
\hline Navsaria $^{13}$ & 2013 & $\begin{array}{l}\text { Vacuum assisted } \\
\text { device }\end{array}$ & $\mathrm{N}$ & Y & $\mathrm{N}$ & $5 \%$ & $65 \%$ & $\begin{array}{c}25 \% \text { Bowel } \\
\text { evisceration, } 40 \% \\
\text { sepsis, } 10 \% \text { bowel } \\
\text { necrosis }\end{array}$ \\
\hline $\begin{array}{l}\text { Steenkamp } \\
\text { et al. }\end{array}$ & 2018 & $\begin{array}{l}\text { Vacuum assisted } \\
\text { mesh mediated } \\
\text { fascial traction }\end{array}$ & Y & Y & $\begin{array}{c}\text { Polypropolene } \\
\text { mesh }\end{array}$ & $0 \%$ & $60 \%$ & $\begin{array}{l}\text { Sepsis } 20 \% \text {, } \\
\text { Mortality } 6 \%\end{array}$ \\
\hline
\end{tabular}


Table 2: Mechanism of injury

\begin{tabular}{llll}
\hline & Blunt trauma - BT (118) & Penetrating trauma - PT (471) & Combination trauma (7) \\
\hline Primary closure & $81(69 \%)$ & $377(80 \%)$ & $5(71 \%)$ \\
Primary TAC & $37(31 \%)$ & $94(20 \%)$ & $2(29 \%)$ \\
Delayed primary closure & $13(35 \%$ of BT TAC $)$ & $36(38 \%$ of PT TAC) & 0 \\
Ventral hernia & $19(51 \%$ of BT TAC $)$ & $28(30 \%$ of PT TAC $)$ & 1 \\
Died TAC & $6(16 \%(1$ died after closure $))$ & $30(32 \%$ of PT TAC) & 1
\end{tabular}

mesh, which is sutured to the fascial edges and gradually tightened. This combines negative pressure with fascial traction. All patients who require TAC are subjected to repeatlaparotomy within 48 hours with the intention of achieving delayed primary closure. If delayed primary closure cannot be achieved, Vacuum Assisted Mesh Mediated Fascial Traction (VAMMFT) is employed at re-laparotomy.

A perforated polyethylene sheet is placed intra-abdominally to cover the viscera and then an oval-shaped polypropylene mesh is sutured to the fascial edges with a continuous monofilament suture. The mesh is run with non-absorbable sutures in a shoe lace type style for future tightening. A thick polyurethane sponge is placed over the mesh and the entire wound is covered with transparent occlusive sheets. The occlusive sheet is then perforated in the middle and linked to a suction device with continuous topical negative pressure. Once the VAMMFT device has been applied, the patient undergoes serial tightening in the ward every 48 hours, using a sterile pack and new vacuum sheets. As soon as sheath approximation is achieved, the patient is scheduled for closure in theatre. At the time of delayed closure in theatre, a lateral release procedure can be performed to ensure a tension free sheath closure. The skin should be loosely approximated with sutures and a vacuum dressing placed across the wound.

Mesh removal is indicated in the following situations:

- In patients whose physiological state precludes resultant increase in intra-abdominal pressure, e.g., poor respiratory efforts or ongoing physiological problems where serial mesh tightening can lead to abdominal compartment syndrome.
- Fistula formation.

- Sheath friability with mesh tearing from tissue and resultant sheath damage.

\section{Statistical Analysis:}

Continuous variables were expressed as mean and standard deviation and were compared with the ANOVA test; categorical data were expressed as proportions and were compared with the Chi-square test. Linear associations were tested with the Pearson's linear correlation model. All the statistical analyses were performed with IBM SPSS 20 (IBM Corp. Released 2011. IBM SPSS Statistics for Windows, Version 20.0. Armonk, NY: IBM Corp).

\section{Results:}

During the four-year period under review a total of 596 patients underwent a laparotomy for trauma. The mean age was 29 years. Of these trauma patients undergoing a trauma laparotomy, $463(78 \%)$ underwent primary closure and 133 $(22 \%)$ required a TAC (Figure 1$)$.

\section{Mechanism of injury:}

In Table 2 the mechanism of injury is divided in penetrating (PT) and blunt trauma (BT). Of all the patients requiring exploratory laparotomy, $471(79 \%)$ sustained penetrating trauma whilst $118(20 \%)$ sustained blunt trauma and $7(1 \%)$ sustained a combination of blunt and penetrating trauma. Of the blunt trauma (BT) group 37 (31\% of all BT) required TAC whilst $96(20 \%$ of all PT) penetrating trauma required TAC.

Table 3: Physiological parameters

\begin{tabular}{|c|c|c|c|c|c|}
\hline & \multicolumn{2}{|c|}{ Primary Closure } & \multicolumn{2}{|c|}{ TAC } & \multirow[t]{2}{*}{ p value } \\
\hline & median & IQR & median & IQR & \\
\hline HR & 93.0 & $(81-188)$ & 110.0 & $(93-123)$ & $<0.001$ \\
\hline $\mathrm{SpO} 2$ & 97.0 & $(95-99)$ & 96.0 & $(92-98)$ & 0,0008 \\
\hline DBP & 73.0 & $(63-83)$ & 67.0 & $(54-80)$ & 0,002 \\
\hline SBP & 122.0 & $(111-134)$ & 113.0 & $(98-128)$ & $<0.001$ \\
\hline $\mathrm{pH}$ & 7.4 & $(7.3-7.4)$ & 7.3 & $(7.2-7.4)$ & $<0.001$ \\
\hline pO2 & 10.3 & $(7.2-12.5)$ & 10.1 & $(5.4-13.6)$ & 0.8 \\
\hline $\mathrm{pCO} 2$ & 5.1 & $(4.5-5.7)$ & 4.9 & $(4.3-5.9)$ & 0.5 \\
\hline $\mathrm{HCO} 3$ & 23.0 & $(20.7-25.1)$ & 19.7 & $(16-23)$ & $<0.001$ \\
\hline lac & 2.2 & $(1.2-3.9)$ & 4.5 & $(2.6-8.0)$ & $<0.001$ \\
\hline
\end{tabular}

HR heart rate, $\mathrm{SpO} 2$ saturation, DBP diastolic blood pressure, SBP systolic blood pressure, pH, pO2 partial oxygen pressure, pCO2 partial carbon dioxide pressure, $\mathrm{HCO} 3$ bicarbonate levels, lac lactate levels 


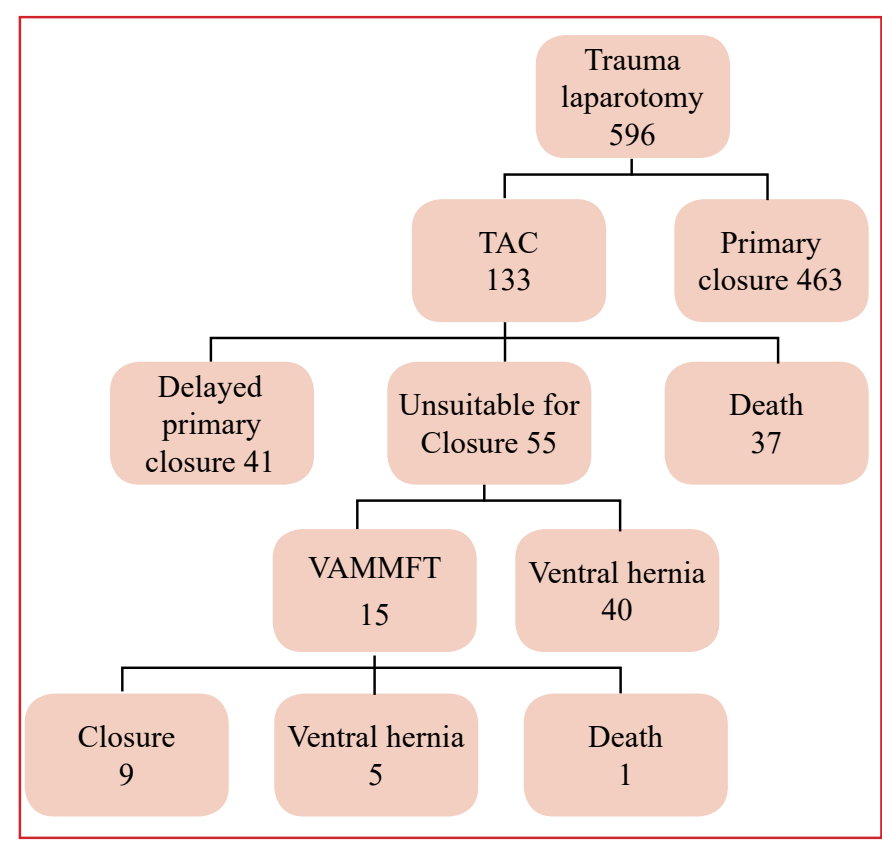

Figure 1: Flow diagram of TAC outcomes

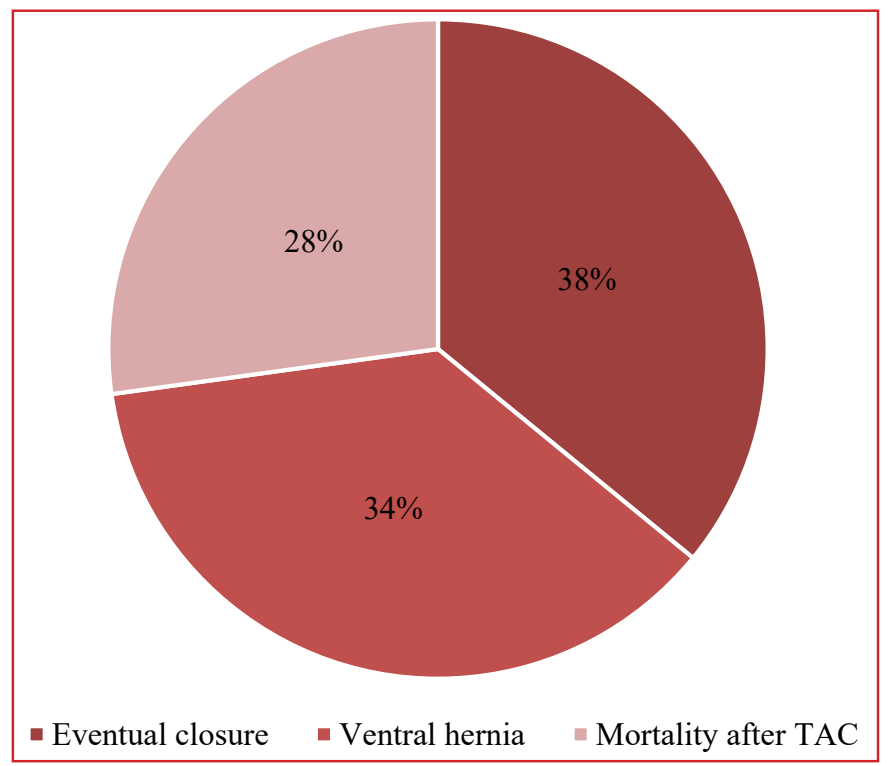

Figure 2: Eventual outcome of patients, divided into death, ventral hernia and eventual closure

\section{Physiological parameters:}

Table 3 compares the presentation physiology of the patients who required TAC and those who did not. By and large patients requiring TAC were significantly more likely to be shocked and acidotic than those who underwent primary closure. Heart rate, lactate and bicarbonate levels were significantly deranged in patients requiring TAC. A total of $36 \%$ of patients requiring TAC were shocked, compared to only $13 \%$ in the primary closure group.

\section{Delayed primary closure:}

In total, 50 patients $(38 \%)$ were discharged with fascial closure having been achieved after primary TAC and
45 patients $(34 \%)$ were discharged from hospital with a ventral hernia. A total of $38(28 \%)$ patients died in hospital. Figure 2 breaks the cohort down by death and by ultimate open abdomen management strategy. There were 41 (31\%) patients in whom delayed primary closure was achieved at repeat laparotomy and there were $55(41 \%)$ in whom this was not possible. Of this cohort of 55 unsuitable patients, as well as some patients that had primary closure but who subsequently required an $\mathrm{OA}$ after repeat-laparotomy, 15 were subjected to the VAMMFT approach.

\section{Vacuum Assisted Mesh Mediated Fascial Traction (VAMMFT):}

VAMMFT yielded a $60 \%$ (9) closure rate in the 15 patients in which it was instituted. The VAMMFT system was inserted on average by day 11 post index laparotomy. By this stage it had become clear that we would be unable to achieve delayed primary closure. Tightening was undertaken every alternative day until the fascial edges were sufficiently approximated to achieve delayed fascial closure. Mesh tightening was performed on average 5 times per patient. It then took a further 12 days of tightening to achieve closure. Each patient ultimately required between 3 to 6 laparotomies to achieve source control and the average length of stay in hospital was 45 days (ranging from 21-103).

\section{VAMMFT Failures:}

Of the 5 VAMMFT failures, intra-abdominal sepsis accounted for 2 and protocol violation a further three. Protocol violation included not inserting the mesh early enough, or removal of the mesh by surgeons not familiar with the technique at repeat laparotomy. In the final protocol, it was determined that mesh insertion should be done at the relaparotomy if delayed primary closure is not possible. Of the failures due to sepsis 1 patient developed superficial wound sepsis and sheath friability before closure and one patient developed deep sepsis with an intra-abdominal pus collection. No enteroatmospheric fistulae developed in this group.

\section{Mortality:}

The overall mortality in this group of patients who underwent a trauma laparotomy was $52(9 \%)$. The mortality rate in those who required a TAC was $37(28 \%)$. A total of 104 patients underwent a damage control procedure. Of these 77 (74\%) required a primary TAC. A total of $34(33 \%)$ of the damage control group died and 24 (23\%) underwent delayed primary closure.

\section{Discussion}

The increased use of damage control principles in trauma and increased awareness of the abdominal compartment syndrome means that surgeons frequently resort to some sort of TAC strategy in the acute management of trauma patients..$^{4-8}$ Table 1 attempts to summarise our experience and compare it with that reported in the literature. Our data shows that TAC is more likely to be needed in patients who are shocked and acidotic and who require damage control surgery. ${ }^{1-3}$ The need 
for a TAC is associated with an increased risk of death and need for ICU as would be expected in a cohort of shocked and acidotic trauma patients. Once TAC has become necessary the spectre of an OA and of ventral hernia is ever present unless delayed closure is possible. Our results suggest that this is only possible in less than fifty percent of patients who require a TAC.

In light of this, strategies need to be implemented to reduce the ventral hernia rate. We cannot afford to use commercial devices on all patients with a TAC and we therefore reserve these devices for patients in whom delayed primary closure cannot be achieved at the first repeat laparotomy. The VAMMFT technique has a $60 \%$ closure rate in our hands and appears to be effective and safe. ${ }^{14,15}$ Our initial experience is encouraging and has helped us refine our algorithms. Ongoing audit will allow us to accrue bigger numbers and to further clarify the role of this technique in the management of these patients.

This study has a number of limitations, many of which bedevil almost all reports on this topic, namely the retrospective nature of the report and the lack of prospective randomisation. This was a new technique introduced in an ad hoc basis for select patients. As confidence with this technique has developed and we have gained experience, it seems likely to become part of our armamentarium.

\section{Conclusion}

Just under one quarter of patients who undergo a trauma laparotomy require TAC. Damage control surgery remains the most common indication for a TAC in trauma. Once TAC is necessary about $40 \%$ of patients will ultimately be left with a ventral hernia. We have adopted a selective VAMMFT strategy to achieve secondary closure. Our initial results with this approach suggest that this is effective and safe.

\section{REFERENCES}

1. Rotondo MF, Schwab CW, McGonigaletal MD. Damage control: an approach for improved survival in exsanguinating penetrating abdominal injury. J Trauma. 1993;35(3):375-83.

2. Saggi BH, Sugerman HJ, Ivantury RR, et al. Abdominal compartment syndrome. J Trauma. 1998;45:597-609.

3. Ivatury RR, Porter JM, Simon RJ, et al. Intra-abdominal hypertension after life threatening abdominal trauma: Prophylaxis, incidence, and clinical relevance to gastric mucosal $\mathrm{pH}$ and abdominal compartment syndrome. J Trauma. 1998;44:1016-23.

4. Ghimenton F, Thomson SR, Muckart DJ, Burrows R. Abdominal content containment: practicalities and outcome. Br J Surg. Jan 2000;87(1):106-9.

5. Quyn AJ, Johnston C, Hall D, Chambers A, Arapova N, Ogston $\mathrm{S}$, Amin A. The open abdomen and temporary abdominal closure systems -historical evolution and systematic review. Colorectal Dis. Aug 2012;14(8):e429-38. doi: 10.1111/j.14631318.2012.03045.x

6. Boele van Hensbroek P, Wind J, Dijkgraaf MGW, Busch ORC, Goslings JC, Goslings CJ. Temporary closure of the open abdomen: a systematic review on delayed primary fascial closure in patients with an open abdomen. World J Surg. 2009;33:199-207.

7. Wittmann DH, Aprahamian C, Bergstein JM. Etappenlavage: advanced diffuse peritonitis managed by planned multiple laparotomies utilizing zippers, slide fastener, and Velcro analogue for temporary abdominal closure. World J Surg. 1990;14:218-26.

8. Wittmann DH, Aprahamian C, Bergstein JM, Edmiston CE, Frantzides CT, Quebbeman EJ, et al. A burr-like device to facilitate temporary abdominal closure in planned multiple laparotomies. Eur J Surg. 1993;159(2):75-9.

9. Brock WB, Barker DE, Burns RP. Temporary closure of open abdominal wounds: the vacuum pack. Am Surg. 1995;61(1):305.

10. Barker DE, Kaufman HJ, Smith LA, Ciraulo DL, Richart CL, Burns, RP. Vacuum pack technique of temporary abdominal closure: a 7-year experience with 112 patients. J Trauma. 2000;48(2):201-7.

11. Smith LA, Barker DE, Chase CW, Somberg LB, Brock WB, Burns RP. Vacuum pack technique of temporary abdominal closure a four-year experience. Am Surg. 1997;63:1102-8.

12. Brock WB, Barker DE, Burns RP. Temporary closure of open abdominal wounds: the vacuum pack. Am Surg. 1995;61:30-5.

13. Navsaria P, Nicol A, Hudson D, Cockwill J, Smith J. Negative pressure wound therapy management of the 'open abdomen' following trauma: a prospective study and systematic review. World J Emerg Surg. 2013;8(1):4.

14. Rasilainen SK, Mentula PJ, Leppäniemi AK. Vacuum and mesh-mediated fascial traction for primary closure of the open abdomen in critically ill surgical patients. Br J Surg. Dec 2012;99(12):1725-32. doi: 10.1002/bjs.8914

15. Petersson U, Acosta S, Björck M. Vacuum-assisted wound closure and mesh-mediated fascial traction - a novel technique for late closure of the open abdomen. World J Surg. 2007;31:21337. 